\title{
Neonatal sepsis in a Nigerian Tertiary Hospital: Clinical features, clinical outcome, aetiology and antibiotic susceptibility pattern
}

\author{
Adediwura O Arowosegbe $^{a *}$, David A Ojo ${ }^{a}$, lyabode O Dedeke ${ }^{b}$, Olufunke B Shittu and Olusola A Akingbade \\ ${ }^{a}$ Department of Microbiology, College of Biosciences, Federal University of Agriculture, Abeokuta, Nigeria \\ ${ }^{b}$ Department of Paediatrics, Federal Medical Centre, Abeokuta, Nigeria \\ 'Department of Medical Microbiology and Parasitology, Federal Medical Centre, Abeokuta, Nigeria \\ *Corresponding author, emails: arowosegbeao@funaab.edu.ng; adearowosegbe@gmail.com
}

Background: Neonatal sepsis is a significant cause of neonatal mortality in developing countries. The aetiological agents and their antimicrobial susceptibility patterns are dynamic.

Objectives: This study determined clinical features, aetiology, antimicrobial susceptibility and clinical outcome of neonatal sepsis in a Nigerian Tertiary Hospital.

Methods: Neonates undergoing sepsis evaluation at a Nigerian Tertiary Hospital were included in the study. Demographic and clinical information were obtained using standard questionnaires. Blood samples were cultured on MacConkey, Blood and Chocolate agar. Isolated bacteria were identified based on morphology, Gram stain appearance and standard commercially prepared biochemical tests. Antimicrobial susceptibility testing was performed on Mueller-Hinton agar using the Kirby-Bauer method.

Results: Eighty-five of the 180 neonates admitted during the study period were recruited. Fifty-five neonates presented with early-onset sepsis and 30 with late-onset sepsis. Culture-proven sepsis was detected in 19 (22.4\%) neonates. The incidence of culture-proven sepsis in the hospital was $2.8 / 100$ live-births. The most common clinical feature at presentation was respiratory distress. Gram-negative bacteria accounted for 78.9 percent of all isolates and were the only organisms encountered in earlyonset sepsis. Isolated pathogens were predominantly Klebsiella spp (31.6\%), Enterobacter spp (21.1\%) and coagulase-negative Staphylococci $(15.8 \%)$. The isolates were most sensitive to ofloxacin. Gram-negative bacteria showed high resistance to cefuroxime and ampicillin. The case-fatality rate was $26 \%$.

Conclusion: Gram-negative bacilli, especially Klebsiella spp, was predominant. Neonatal sepsis persists as a cause of mortality in this region. Regular antimicrobial surveillance for empirical treatment remains an important component of neonatal care.

Keywords: antimicrobial susceptibility, mortality, neonatal sepsis, outcome

\section{Background}

Globally, about forty percent of under-five deaths occur in the neonatal period resulting in 2.9 million newborn deaths each year. ${ }^{1}$ The highest mortality rates for newborns are found in the poorest countries and a third of these deaths are attributed to infections acquired by the baby during labour and delivery or after birth. ${ }^{1}$ Nigeria accounts for the highest number of neonatal deaths in Africa and third in the world (after India and China) with sepsis responsible for about $30 \%$ to $50 \%$ of deaths. ${ }^{2}$ In Nigeria, the prevalence of neonatal sepsis reported from previous hospital-based studies ranges between 7.04 and 22.9 per 1000 live births. ${ }^{3}$ Also, mortality rates from neonatal sepsis have ranged from 26.7\% in Abakaliki, to 32.2\% in Sagamu and 33.3\% in lle-Ife, over the last two decades. ${ }^{4}$

The term neonatal sepsis, refers to systemic infection of neonates including septicaemia, pneumonia, meningitis, arthritis, osteomyelitis, and urinary tract infection. ${ }^{5}$ It is a clinical syndrome characterised by systemic signs of circulatory compromise (e.g., poor peripheral perfusion, pallor, hypotonia, poor responsiveness). ${ }^{6}$ Neonatal sepsis could be from early onset, within the first $72 \mathrm{~h}$ of life, and presumed to be acquired through prenatal and intrapartum maternal transmission, or late onset from the fourth day to fourth week of life. ${ }^{5}$

Sepsis-related case fatality rates are largely preventable with proper antimicrobial use and aggressive supportive care.
However, neonatal sepsis has no pathognomonic features and the clinical presentation varies as well. ${ }^{5}$ Poor or delayed laboratory services also make laboratory diagnosis difficult in resource poor settings. As a result, neonatal healthcare providers in resource limited settings make tentative diagnosis and empirical treatment of neonatal sepsis especially using the new neonatal WHO International Management of guidelines. ${ }^{6}$

However, the diversity of organism causing neonatal sepsis varies significantly across different regions and changes over time, even in the same place. This variation may affect the success of empirical management. ${ }^{6}$ In developed countries, the most common causes of neonatal sepsis are Group B streptococci (GBS), Escherichia coli and Listeria monocytogenes while Gramnegative bacteria and coagulase-negative staphylococci are the most common in developing countries. ${ }^{7}$ It is noteworthy that the growing incidence of drug resistant bacterial isolates has also made treatment more difficult and costly. ${ }^{8}$

It is imperative, therefore, that the epidemiology of neonatal sepsis should be regularly updated to provide information required for regular review of the choice of drugs most suitable for the treatment of neonatal sepsis in different places and at different times. ${ }^{9}$ This study was therefore conducted to determine the clinical presentation, etiological agent of sepsis, and antibiotic sensitivity pattern in neonates with suspected septicaemia admitted in the neonatal unit of a Tertiary Hospital in Nigeria. 


\section{Materials and methods}

\section{Study location and design}

This was a prospective cross-sectional study of neonates presenting with sepsis at the Special Care Baby Unit (SCBU) of Federal Medical Centre, Abeokuta, Nigeria. The hospital serves the health needs of the people of the main city and surrounding towns, and is the main referral centre providing neonatal care for these communities. The study was carried out between January 2013 and April 2013.

In the unit, blood culture was routinely performed for babies with risk factors for sepsis or clinical features suggestive of sepsis. After blood sample collection, the neonates commenced empirical antibiotic therapy with cefuroxime and gentamicin. ${ }^{10}$ Treatment is reviewed based on outcome of blood culture results. For positive cultures, antibiotics were continued for at least 10 days according to blood culture results.

\section{Study population}

All the neonates (0-28 days) with risk factors and/or clinical signs and symptoms of sepsis at the time of admission, or who developed sepsis during their hospital stay, were assessed using the WHO case definition for neonatal septicaemia and, hence, included in the study. For this study, neonatal septicaemia was defined as systemic bacterial infection in a neonate with positive blood culture within the first 28 days of life. Neonatal sepsis was categorised according to the infant age at the onset of symptoms into: early-onset sepsis ( $\leq 72 \mathrm{~h}$ ) and late-onset sepsis ( $72 \mathrm{~h}) .^{5}$

Neonates with prior antibiotics use were excluded to minimise interferences with laboratory results. Neonates delivered at the hospital were designated 'in-born' babies, while babies referred to the unit after delivery outside the hospital were designated 'out-born' babies. A duration of rupture of foetal membranes greater than $24 \mathrm{~h}$ prior to delivery was defined as 'prolonged rupture of membrane', and a duration of active labour greater than $12 \mathrm{~h}$ was defined 'prolonged labour'.

Socio-demographic characteristics, neonatal details (clinical features, sex, age at admission, weight at admission, estimated gestational age (EGA) at delivery, place of birth) and details of perinatal events (including occurrence of prolonged rupture duration of fetal membrane, duration of labour, occurrence of pyrexia during labour, mode of delivery, place of antenatal care, and place of delivery) were obtained with the use of a study proforma designed for this study. The socio-economic status of the parents was assessed using the method recommended by Ogunlesi, ${ }^{4}$ which is based on the occupation of the father and the educational qualification of the mother.

The outcome of hospitalisation was recorded as 'discharge in good condition', 'discharge against medical advice', and death.

\section{Ethical clearance and permission}

An ethical clearance certificate was obtained from the Research and Ethical Clearance Committee of the hospital. For each baby, a written informed consent was also obtained from the parent(s) or guardians, as necessary.

\section{Sample collection and processing}

Blood was collected from a peripheral vein on the dorsum hand by the doctor on duty (already informed about the protocol of the study) after thoroughly cleansing the patient's skin with an alcohol swab and allowing the skin to dry before taking blood.
Approximately $1 \mathrm{ml}$ of blood was inoculated directly into each of the two bottles containing blood culture media (Brain heart infusion and thioglycolate broth). The blood cultures were incubated at $37^{\circ} \mathrm{C}$ for seven days and observed daily for visible growth of micro-organisms by one of the following: gas production (presence of air bubbles); haemolysis; and, broth coagulation. Subcultures were done on the third, fifth and seventh day on selective and enriched media including Blood, Chocolate and MacConkey agar.

The agar plates were incubated under aerobic and anaerobic conditions. Colonies on solid agar plates were identified based on characteristic morphology, Gram stain appearance and standard commercially prepared biochemical tests. Susceptibilities to common antibiotics were determined by the disk-diffusion method. ${ }^{11}$ Blood culture reports were collected and analysed for the frequency of different bacterial isolates and their antibiotic sensitivity pattern. Isolates showing an intermediate level of sensitivity were classified as resistant.

\section{Data analysis}

Statistical Package for Social Sciences version 17.0 was used for data entering and analysis. Data was described using standard methods. Values are expressed as mean \pm standard deviation (SD), unless otherwise stated. Mortality and case-fatality rates were recorded and expressed as percentages.

\section{Results}

\section{Neonatal and maternal characteristics}

The total number of live births in the hospital during the study period was 327. There were 180 admissions, and 85 (47.2\%) participants had suspected sepsis based on clinical features and risk factors. The male to female ratio was 1:1.1. Fifty-four babies (63.5\%) had early-onset sepsis and thirty-one (36.5\%) had lateonset sepsis. Majority of the neonates belonged to the lower and middle socio-economic class. Mean age at admission was $16.28 \pm 2.61 \mathrm{~h}$ and $8.25 \pm 1.00$ days for neonates with EOS and LOS, respectively; while, the mean weight was $2.5 \pm 0.8 \mathrm{~kg}$. Of the 85 neonates, 28 (32.9\%) were in-born babies, while $57(67.1 \%)$ were out-born babies and were referred to the hospital. Eleven $(19.3 \%)$ of these out-born babies were born at home or at traditional birth centres, $21(36.8 \%)$ in private nursing homes and $25(43.9 \%)$ in hospitals or clinics. Thirty-two (37.6\%) of the 85 neonates were preterm. About two-third (69.4\%) of the neonates were delivered vaginally and the remainder were delivered by Caesarean section. Of the 85 neonates, 25 (29.4\%) were born after prolonged rupture of membranes and 18 (18.8\%) were born after prolonged labour (Table 1).

\section{Clinical characteristics of participants}

Most frequently reported clinical features of participants included respiratory distress (46\%), refusal of feed (27\%), convulsion (25\%) and fever (25\%). Others were weak cry (13\%), abdominal distension (5\%), high pitched cry/irritability (5\%), apnea (5\%), and pallor (4\%). The most common clinical features among babies with sepsis were respiratory distress (53\%), fever (32\%), refusal of feed (26\%), and convulsion (26\%) (Figure 1).

\section{Bacterial isolation}

Nineteen (22.4\%) of 85 participants had positive blood culture. There was a $22.4 \%$ prevalence of culture-proven sepsis among both in-born and out-born neonates admitted in the neonatal unit with suspected sepsis. Thirteen $(24.1 \%)$ of the 54 patients with EOS and 6 (19.4\%) of the 31 patients with LOS had positive 
Table 1: Maternal and neonatal characteristics of the study population.

\begin{tabular}{|c|c|c|}
\hline Characteristics & Variables & Frequency (\%) \\
\hline \multicolumn{3}{|l|}{ (I) Maternal data } \\
\hline \multirow[t]{4}{*}{ Maternal age } & $<20$ & $04(4.7)$ \\
\hline & $20-29$ & $32(37.7)$ \\
\hline & $30-39$ & $42(49.4)$ \\
\hline & $>40$ & $07(8.2)$ \\
\hline \multirow[t]{3}{*}{ Socio- economic class } & High & $21(24.7)$ \\
\hline & Middle & $26(30.6)$ \\
\hline & Low & $28(44.9)$ \\
\hline \multirow[t]{2}{*}{ Booking status } & Booked & $27(31.8)$ \\
\hline & Unbooked & $58(68.2)$ \\
\hline \multirow[t]{3}{*}{ Place of delivery } & Hospital & $53(62.4)$ \\
\hline & Private Nursing Homes & $21(24.7)$ \\
\hline & Home/TBA & $11(12.9)$ \\
\hline \multirow[t]{2}{*}{ Mode of delivery } & Vaginal Delivery & $58(68.2)$ \\
\hline & Ceaserean Section & $26(30.6)$ \\
\hline \multirow[t]{3}{*}{ Parity } & Primiparous & $32(37.7)$ \\
\hline & Multiparous & $42(49.4)$ \\
\hline & Grandmultiparous & $11(12.9)$ \\
\hline \multicolumn{3}{|l|}{ (II) Neonatal data } \\
\hline \multirow[t]{2}{*}{ Sex } & Male & $40(47.1)$ \\
\hline & Female & $45(52.9)$ \\
\hline \multirow[t]{2}{*}{ Age } & $<3$ days & $54(63.5)$ \\
\hline & $>3$ days & $31(36.5)$ \\
\hline \multirow[t]{2}{*}{ Birth weight/g } & Low Birth weight (<2500 g) & $39(45.9)$ \\
\hline & $\begin{array}{l}\text { Normal Birth weight } \\
\qquad(>2500 \mathrm{~g})\end{array}$ & $46(54.1)$ \\
\hline \multirow[t]{2}{*}{ Estimated gestational age } & $<37$ weeks (Preterm) & $32(37.6)$ \\
\hline & >37 weeks (Term) & $53(62.4)$ \\
\hline \multirow[t]{2}{*}{ Outcome of management* } & Discharge & $48(64)$ \\
\hline & Death & $27(36)$ \\
\hline
\end{tabular}

*Ten of the neonates were discharged against medical advice and were excluded.

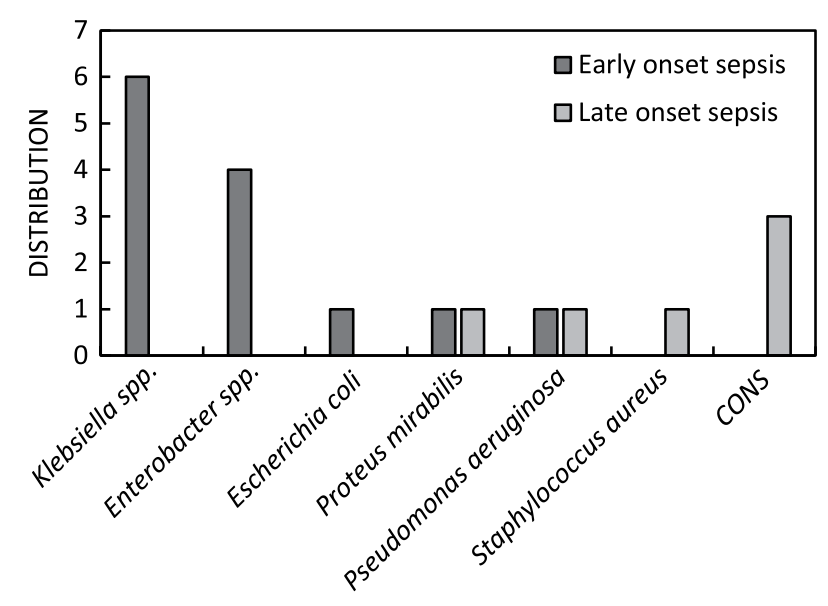

Figure 1: Distribution of organisms according to onset of sepsis.

blood cultures. Of the 85 neonates with suspected sepsis, only 27 were in-born. Nine of these in-born neonates had culture-proven sepsis and 19 had clinical sepsis. The incidence of culture-proven and clinical sepsis in the hospital (among all 327 in-born patients) during the study period was $2.8 \%$ and $5.5 \%$, respectively.

Gram-negative bacilli were the only organisms encountered in the first $72 \mathrm{~h}$, with Klebsiella spp accounting for almost half (46.2\%) of all the isolates encountered during this period. Grampositive cocci accounted for two-thirds of the organism encountered after $72 \mathrm{~h}$ of life (Figure 1).

\section{Antibiotic sensitivity patterns}

The sensitivity pattern showed that Gram-negative organisms had a high degree of resistance to commonly used antibiotics (Table 2). These isolates were most sensitive to ofloxacin and had the highest resistance to ampicillin. In the Gram-negative group, the best overall sensitivity was to ofloxacin and Cefepime (86.4\%), while highest resistance was to cefuroxime, ampicillin, and erythromycin (92.3\%). As a group, the Gram-positive organisms had $100 \%$ sensitivity to cefuroxime, cefepime, ceftriaxone, ofloxacin, and gentamycin.

\section{Clinical outcome of management}

Ten (11.8\%) of the neonates recruited into the study, including 2 with positive blood culture, were discharged against medical advice. Forty-eight (56.5\%) of the neonates were discharged in good condition after appropriate interventions were administered, including antibiotic therapies. Death occurred in $27(31.8 \%)$ of the 85 neonates. Five $(26.3 \%)$ of the neonates with positive blood culture died. The case-fatality rate was $26 \%$.

\section{Discussion}

The findings of this study show that neonatal sepsis is common and contributes to mortality among neonates admitted in the neonatal ward at a Nigerian Tertiary Hospital. The prevalence of positive blood culture sepsis in this study was $22.4 \%$. This is similar with reports from studies in Nigeria and other developing countries, ${ }^{12-14}$ which were also hospital-based and cross-sectional studies. However, the prevalence from this study was much higher than reports from the WHO Young Infants Study Group from four different countries, with rates of positive blood culture ranging from $4 \%$ in the Philippines and $5 \%$ in Papua New Guinea (PNG) to $10 \%$ in Ethiopia and $11 \%$ in Gambia. ${ }^{15}$ This difference can be attributed to the presence of more neonates with high risk for sepsis presenting in hospitals compared to numbers found in community settings in the WHO Young Infants Study.

The case-fatality rate observed in the present study was comparable to reports from other studies in Nigeria., ${ }^{415}$ In contrast, very low rates of prevalence and case fatalities were reported in the developed countries, which can be explained by the high quality of life and high standard of health care and hospital services in these countries. ${ }^{3}$

In this study, early-onset sepsis was more common than lateonset sepsis, which is in agreement with the reports from other developing countries, including Cameroon (77.5\%) ${ }^{16}$ and Bangladesh (70.7\%), ${ }^{17}$ but in contrast with other reports from Pakistan $(42 \%)^{18}$ and Libya (31\%), ${ }^{19}$ where late-onset sepsis was more common.

Gram-negative bacteria were responsible for most of the cases of early-onset neonatal sepsis and Gram-positive bacteria for late onset sepsis. This is consistent with reports from other developing countries, including Nigeria. ${ }^{13,20-21}$ Reports from a review of 
Table 2: Bacterial isolates and their antibiotic sensitivity pattern.

\begin{tabular}{|c|c|c|c|c|c|c|c|c|c|}
\hline \multicolumn{10}{|l|}{ Antimicrobial agents (\%) } \\
\hline Isolates & & CRX & CPM & CXM & AMP & OFL & CPR & GEN & CAZ \\
\hline \multirow[t]{2}{*}{ Klebsiella spp } & $S$ & $0(0)$ & $2(33)$ & $0(0)$ & $1(17)$ & $5(83)$ & $4(67)$ & $2(33)$ & 2(33) \\
\hline & $\mathrm{R}$ & $6(100)$ & $4(67)$ & $6(100)$ & $5(83)$ & $1(17)$ & $2(33)$ & $4(67)$ & $4(67)$ \\
\hline \multirow[t]{2}{*}{ Enterobacter spp } & $S$ & $3(75)$ & $4(100)$ & $0(0)$ & $0(0)$ & $4(100)$ & $2(50)$ & $1(25)$ & $0(0)$ \\
\hline & $\mathrm{R}$ & $1(25)$ & $0(0)$ & $4(100)$ & $4(100)$ & $0(0)$ & $2(50)$ & $3(75)$ & $4(100)$ \\
\hline \multirow[t]{2}{*}{ Proteus mirabilis } & $S$ & $1(50)$ & $2(100)$ & $0(0)$ & $0(0)$ & $2(100)$ & $2(100)$ & $1(50)$ & $0(0)$ \\
\hline & $\mathrm{R}$ & $1(50)$ & $0(0)$ & $2(100)$ & $2(100)$ & $0(0)$ & $0(0)$ & $1(50)$ & $2(100)$ \\
\hline \multirow[t]{2}{*}{ Pseudomonas aeruginosa } & $S$ & $1(50)$ & $2(100)$ & $1(50)$ & $0(0)$ & $1(50)$ & $0(0)$ & $2(100)$ & $0(0)$ \\
\hline & $\mathrm{R}$ & $1(50)$ & $0(0)$ & $1(50)$ & $2(100)$ & $1(50)$ & $2(100)$ & $0(0)$ & $2(100)$ \\
\hline \multirow[t]{2}{*}{ Escherichia coli } & $S$ & $0(0)$ & $1(100)$ & $0(0)$ & $0(0)$ & $1(100)$ & $1(100)$ & $0(0)$ & $0(0)$ \\
\hline & $\mathrm{R}$ & $1(100)$ & $0(0)$ & $1(100)$ & $1(100)$ & $0(0)$ & $0(0)$ & $1(100)$ & $1(100)$ \\
\hline \multirow[t]{2}{*}{ Staphylococcus aureus } & $S$ & $1(100)$ & $1(100)$ & $1(100)$ & $0(0)$ & $1(100)$ & $1(100)$ & $1(100)$ & $0(0)$ \\
\hline & $\mathrm{R}$ & $0(0)$ & $0(0)$ & $0(0)$ & $1(100)$ & $0(0)$ & $0(0)$ & $0(0)$ & $1(100)$ \\
\hline \multirow[t]{2}{*}{ Coagulase-negative staphylococci } & $S$ & $3(100)$ & $3(100)$ & $2(67)$ & $3(100)$ & $3(100)$ & $3(100)$ & $3(100)$ & $1(33)$ \\
\hline & $\mathrm{R}$ & $0(0)$ & $0(0)$ & $1(33)$ & $0(0)$ & $0(0)$ & $0(0)$ & $0(0)$ & $2(67)$ \\
\hline
\end{tabular}

Notes: KEY: S - Sensitive, R - Resistant Ceftriaxone (CRX), Cefepime (CPM), Cefuroxime (CXM), Ampicillin (AMP), Ofloxacin (OFL), 0(GEN), Ceftazidime (CAZ).

neonatal sepsis in 19 developing countries have shown that more than half of the cases of neonatal sepsis were due to Staphylococcus aureus, E. coli or Klebsiella spp. ${ }^{22}$ Other studies in Malawi have reported GBS as the most common pathogen causing neonatal sepsis..$^{23,24}$ The organisms isolated with respect to time of onset in this study seem to fit with the proposed sources of bacteria in neonatal sepsis: Gram-negative bacteria were the predominant isolates in the birth canal for early-onset sepsis, and Gram-positive bacteria were predominantly isolated from hospital or community sources for late sepsis. ${ }^{6,25}$

In this study, respiratory distress, convulsions, fever, and refusal to feed were the most frequent clinical manifestation (signs and symptoms) for admission. In a similar study, ${ }^{26}$ respiratory distress (31.3\%) was the major presenting feature, while other studies 3,16 have reported fever, refusal to feed, respiratory distress, poor activity and neonatal jaundice as the most common symptoms. The variance in clinical features of neonatal sepsis further gives credence to the nonspecific nature of its manifestations and the need for a high index of suspicion. In this study, a very high number of discharges against medical advice were recorded. This is in line with similar reports on paediatric and neonatal discharges against medical advice across Nigeria. ${ }^{27,28}$ Various social and economic factors are responsible for the high rates of discharges against medical advice in Nigeria. This includes lack of funds, perceived improvement, lack of improvement, inconvienience, and religion.

The high degree of resistance Gram-negative organisms exhibited to commonly-used antibiotics, predominantly to broad-spectrum cephalosporins, reported in this study was comparable with other studies. ${ }^{16}$ Reports of multi-resistant bacteria causing neonatal sepsis in developing countries are increasing, particularly in intensive care units. ${ }^{26}$ Downie et al. ${ }^{23}$ reported that more than $40 \%$ of sepsis in neonates was due to pathogens that were resistant (or had reduced susceptibility) to the antibiotic combination of ampicillin/penicillin and gentamicin, or the increasingly used alternative, third-generation cephalosporins. These reports buttress the need for the continuous review of empirical antibiotics used in the treatment of neonatal sepsis to ensure optimal antimicrobial use.

The isolates were most sensitive to ofloxacin and cefepime. Awoniyi et al. ${ }^{29}$ also reported that neonatal sepsis isolates were most sensitive to ofloxacin. About two-thirds of the isolates were sensitive to ceftriaxone, although higher rates of sensitivity to ceftriaxone have been reported in other studies. ${ }^{3,12}$ There was a high resistance to ampicillin as reported in other studies. ${ }^{16,29}$ This high resistance rate against ampicillin was not surprising. The indiscriminate use of antibiotics in Nigeria might account for the high resistance rates observed. ${ }^{27}$

\section{Conclusion}

The most common micro-organisms causing neonatal sepsis in EOS were Gram-negative bacilli, particularly Klebsiella spp and coagulase-negative Staphylococci in LOS. There was an exceedingly high rate of resistance to ampicillin and cephalosporin for all micro-organisms.

\section{Recommendation}

Guidelines on the reduction of emergence of drug resistance must be provided and implemented in new-born units.

Author's Contribution - Concept: Arowosegbe AO. Design: Arowosegbe AO, Dedeke, IOF, Ojo DA, Shittu,OB, Akingbade, AO. Data collection, analysis and interpretation: Arowosgebe, $A O$, Dedeke, IOF, Akingbade OA. Manuscript preparation: Arowosegbe, AO. Reviewing of manuscript: Ojo, DA, Shitttu,OB, Dedeke, IOF. All authors read and approved the final manuscript.

\section{References}

1. Save the Children. Ending newborn deaths: ensuring every baby survives. London: Save the Children; 2014.

2. Ahman E, Zupan J. Neonatal and perinatal mortality: country, regional and global estimates 2004. Geneva: World Health Organization; 2007

3. Ogundare EO, Akintayo AA, Dedeke IOF, et al. Neonatal septicaemia in a rural nigerian hospital: aetiology, presentation and antibiotic sensitivity pattern. Br J Med Med Res. 2016;12(7):1-11. https://doi. org/10.9734/BJMMR 
4. Ogunlesi TA, Ogunfowora OB. Predictors of mortality in neonatal septicaemia in an underresourced setting. J Natl Med Assoc. 2010;102(10):915-22. https://doi.org/10.1016/S0027-9684(15)30710-0

5. Shalini TS, Malik GK. Neonatal Sepsis: past, present and future; a review article. Inter J Med Up. 2010;5(2):45-54.

6. Edmund K, Zaidi A. New approaches to preventing, diagnosing and treating neonatal sepsis. PloS Med. 2010;7(3):1-8.

7. Palazzi, D, Klein, J, Baker, C. Bacterial sepsis and meningitis.In: Remmington JS, Klein J, edtiors. Infectious diseases of the fetus and newborn infant. 6th ed. Philadelphia: Elsevier Saunders. 2006. p. 24795. https://doi.org/10.1016/B0-72-160537-0/50008-6

8. Roy I, Jain A, Kumar M, Agarwal SK. Bacteriology of neonatal septicaemia in a tertiary care hospital of northern India. Indian J Med Microbiol. 2002;20:156-9.

9. Shittu OB, Akpan I, Popoola TO, et al. Epidemiological features of a GIS-supported investigation of cholera outbreak in Nigeria. J Pub Health and Epi. 2010;2(5):152-62.

10. Coffey P, Kelly K, Baqui A, et al. Injectable Antibiotics for Treatment of Newborn Sepsis. Prepared for the United Nations Commission on Commodities for Women's and Children's Health; 2012.

11. Clinical and Laboratory Standard Institute (CLSI). Performance standards for antimicrobial susceptibility testing. Nineteenth informational supplement M100-S19. Wayne: Clinical and Laboratory Standard Institute. 2009.

12. Adeleke SI, Belonwu RO. Bacterial isolates in neonatal septicaemia in Kano. Nigeria. Pinnacle Int. J. Med. Sci. 2006;1(1):17-20.

13. Kayange N, Kamugisha E, Mwizamholya DL, et al. Predictors of positive blood culture and death among neonates with suspected neonatal sepsis in a tertiary hospital. Mwanza-Tanzania. BMCPediatr.2010;10:39. https://doi.org/10.1186/1471-2431-10-39

14. Ojukwu JU, Abonyi LE, Ugwu J, et al. Neonatal septicaemia in high risk babies in Eastern Nigeria. J Perinatal Med. 2006;34:166-72.

15. WHO young infant study group. Bacterial etiology of serious infection in young infants in developing countries: results of multicenter study. Pediatr. Dis. J. 1999;18:S17-22.

16. Chiabi A, Djoupomb M, Mah E, et al. The clinical and bacteriogical spectrum of neonatal sepsis in a tertiary hospital in Yaounde. Cameroon. Iran J Pediatr. 2011;21(4):441-8.

17. Rasul CH, Hassan MA, Habibullah M. Neonatal sepsis and use of antibiotic in tertiary care hospital. Pak J Med Sci. 2007;23:78-81.
18. Aftab R, lqbal I. Bacteriological agents of neonatal sepsis in NICU at Nishtar Hospital Multan. J Coll Physicians Surg Pak. 2006;16:216-9.

19. Misallati A, el-Bargathy S, Shembesh N. Blood-culture-proven neonatal septicaemia: a review of 36 cases. East Mediterr Health J. 2000;6:483-6.

20. Kayange N, Kamugisha E, Mwizamholya DL, Jeremiah S, Mshana SE. Predictors of positive blood culture and death among neonates with suspected neonatal sepsis in a tertiary hospital. Mwanza-Tanzania BMC Pediatr. 2010;10:39. https://doi.org/10.1186/1471-2431-10-39

21. Mokuolu AO, Jiya N, Adesiyun OO. Neonatal septicaemia in Ilorin: bacteria pathogens and antibiotic sensitivity pattern. Afr J Med Med Sci. 2002;31:127-30.

22. Downie L, Armiento R, Subhi R, et al. Community-acquired neonatal and infant sepsis in developing countries: efficacy of WHO's currently recommended antibiotics-systematic review and meta-analysis. Arch Dis Child. 2012;1-9.

23. Milledge J, Calis JCJ, Graham SM, et al. Aetiology of neonatal sepsis in Blantyre, Malawi: 1996-2001. Ann Trop Paediatr. 2005;25:101-10. https://doi.org/10.1179/146532805X45692

24. Gray KJ, Beneth SL, French N, Phiri AJ, Graham SM. Invasive group B streptococcal infection in infants, Malawi. Emerg Infect Dis 2007;13:223e9.

25. Newton O, English M. Young infant sepsis: aetiology, antibiotic susceptibilityand clinical signs.Trans RSocTropMed Hyg.2007;101(104):959-66. https://doi.org/10.1016/j.trstmh.2007.05.005

26. Karthikeyan G, Premkumar K. Neonatal sepsis: Staphylococcus aureus as the predominant pathogen. Indian J Pediatr. 2001;68:715-7. https://doi.org/10.1007/BF02752407

27. Opara P, Eke G. Discharge against medical advice among neonates admitted into a Special Caare Baby Unit in Port-Harcourt Nigeria. Inter J Pediatr Neonatol. 2010;12(2):1-725.

28. Onyinuka AN. Pediatric discharge gainst medical advice : Experience from a Nigerian Secondary Healthcare Institution. Med J Islamic Republic of Iran. 2011;25(4):194-9.

29. Awoniyi DO, Udo SJ, Oguntibeju OO. An epidemiological survey of neonatal sepsis in a hospital in western Nigeria. Afr J Microbiol Res. 2009;3(7):385-9.

Received: 01-02-2017 Accepted: 25-05-2017 